\title{
Long-lived memory for mesoscopic quantum bits
}

\author{
J.M. Taylor, C.M. Marcus, and M.D. Lukin \\ Department of Physics, Harvard University, Cambridge, Massachusetts 02138
}

(Dated: October 31, 2018)

\begin{abstract}
We describe a technique to create long-lived quantum memory for quantum bits in mesoscopic systems. Specifically we show that electronic spin coherence can be reversibly mapped onto the collective state of the surrounding nuclei. The coherent transfer can be efficient and fast and it can be used, when combined with standard resonance techniques, to reversibly store coherent superpositions on the time scale of seconds. This method can also allow for "engineering" entangled states of nuclear ensembles and efficiently manipulating the stored states. We investigate the feasibility of this method through a detailed analysis of the coherence properties of the system.
\end{abstract}

PACS numbers: 73.21.La, 76.70.-r, 03.67

A broad effort is now underway to develop new techniques for coherently controlling quantum degrees of freedom in mesoscopic systems [1]. These efforts are stimulated in part by the proposals to use these systems as quantum bits in the context of quantum information science. The fast decoherence associated with solid-state environments proves to be the main obstacle for experimental realization of such control.

Spin degrees of freedom of electrons confined in semiconductor quantum dots are attractive qubit candidates [2, 3]. Relatively long decoherence times are expected for such systems and techniques for the coherent manipulation and measurement of electron spins are now being developed. For the latter, coupling of spin and charge degrees of freedom is probably necessary. Experimental measurements of the spin relaxation times indicate sub- $\mathrm{MHz}$ rates [4], although it is not yet clear what will determine the ultimate coherence lifetimes.

This Letter describes a technique for greatly extending the lifetimes of electron-spin qubits in confined structures by coherently mapping an arbitrary spin superposition state into the spins of proximal, polarized nuclei. This is achieved by effective control of the spin-exchange part of hyperfine contact interaction. After the transfer is completed, the resulting superpositions could be stored for a very long time - up to seconds - and mapped back into the electron spin degrees of freedom on demand. We further show that the stored states can be manipulated using an extension of standard resonance techniques.

Although it is widely known that nuclear spins can possess exceptionally long coherence times due to their weak environmental coupling, single nuclear spins are very difficult to manipulate and measure in practice [5]. In the present approach these problems are circumvented by using collective nuclear degrees of freedom, which do not require individual addressing and control. We demonstrate that such collective states are extremely robust with respect to realistic imperfections, such as partial initial polarization and spin diffusion, and decoherence As a result the present technique allows combines the strengths of electron spin (or charge) manipulation with the excellent long-term memory provided by nuclei.

When uncontrolled, the coupling of electronic spin degrees of freedom to nuclei may be considered an environmental decoherence process. Interesting features of this process arise from its non-Markovian nature [6, 7, 8]. The present paper shows that properly controlled coupling of electrons to nuclei can be used to greatly extend the effective coherence time of electron spins. This study parallels recent work involving the use of atomic ensembles as quantum information carriers [9].

To illustrate the technique, consider a single electron localized in quantum dot. The effective Hamiltonian for the electron and $N$ spin- $I_{0}$ nuclei in a magnetic field $B_{0}$ along the $z$-axis is

$$
\hat{H}=g^{*} \mu_{B} B_{0} \hat{S}_{z}+g_{n} \mu_{n} B_{0} \sum_{j} \hat{I}_{z}^{j}+\hat{V}_{H F} ;
$$

The first two terms of Eq. 1 correspond to the Zeeman energy of the electronic and nuclear spins; the third term is the hyperfine contact interaction between the $s$-state conduction electrons and the nuclei in the dot, $\hat{V}_{H F}=\sum_{j} a_{j} \hat{\overrightarrow{I^{j}}} \cdot \hat{\vec{S}}$. The coefficients $a_{j}=A v_{0}\left|\psi\left(\vec{r}_{j}\right)\right|^{2}$ correspond to the one-electron hyperfine interaction with the nuclear spin at site $\vec{r}_{j}$, where $A$ is the hyperfine contact interaction constant, $v_{0}$ is the volume of a unit cell, and $\psi(\vec{r})$ is the envelope function of the localized electron. The hyperfine term can be written $\hat{V}_{H F}=\hat{V}_{D}+\hat{V}_{\Omega}$ where $\hat{V}_{D}=\sum_{j} a_{j} \hat{I}_{z}^{j} \hat{S}_{z}$ and $\hat{V}_{\Omega}=\sum_{j} a_{j} / 2\left(\hat{I}_{-}^{j} \hat{S}_{+}+\hat{I}_{+}^{j} \hat{S}_{-}\right)$. $\hat{V}_{D}$ produces an effective magnetic field for the electron $\hat{B}_{\text {eff }}=B_{0}-1 / g^{*} \mu_{B} \sum_{j} a_{j} \hat{I}_{z}^{j}$, which results in the wellknown Overhauser shift. However, when $g^{*} \mu_{B} \hat{B}_{\text {eff }} \ll \hat{V}_{\Omega}$, spin exchange becomes the dominate effect.

We start with a perfectly polarized nuclear ensemble $|\mathbf{0}\rangle_{n}=\left|I_{0}, \ldots, I_{0}\right\rangle_{n}$, as shown in Fig. 1(a). Due to a total angular momentum conservation only a spin-down initial electron state can undergo non-trivial evolution. When the dynamics are governed by $\hat{V}_{\Omega}\left(\hat{B}_{\text {eff }} \rightarrow 0\right)$, there is a coherent exchange of excitations between electronic and nuclear degrees of freedom. For the inital state $|\downarrow\rangle_{e} \otimes|\mathbf{0}\rangle_{n}$, spin-exchange couples this state to the collective nuclear 
(a)

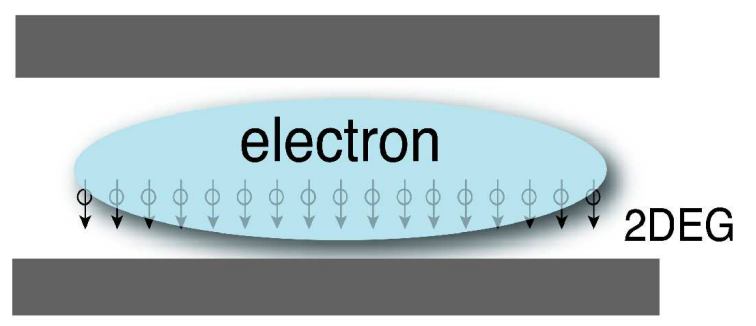

(b)

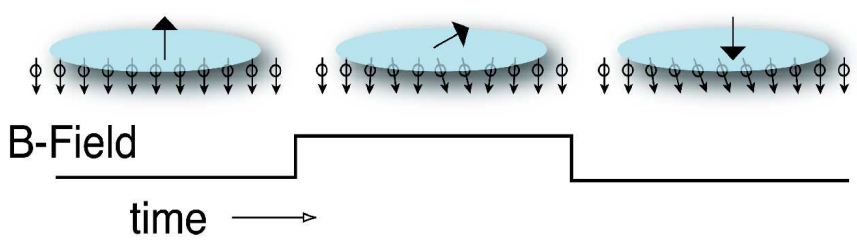

FIG. 1: (a) A schematic of an electron trapped in a quantum dot with polarized nuclei. (b) Storage, where a pulse in the magnetic field starts amd brings the spins into resonance. After the electron spin has flipped ( $\pi$-pulse) the pulse is ended. Readout is the same process in reverse.

excitation with one flipped spin $|\uparrow\rangle_{e} \otimes|\mathbf{1}\rangle_{n}$ with

$$
|\mathbf{1}\rangle_{n} \equiv\left(\sum_{j}\left|a_{j}\right|^{2}\right)^{-1 / 2} \sum_{j} a_{j}\left|I_{0}, \ldots,\left(I_{0}-1\right)_{j}, \ldots, I_{0}\right\rangle_{n} .
$$

Hence the evolution is given by the two-level dynamics

$$
\left[\begin{array}{l}
|\downarrow\rangle|\mathbf{0}\rangle \\
|\uparrow\rangle|\mathbf{1}\rangle
\end{array}\right](t)=\left[\begin{array}{cc}
\cos (\Omega t / 2) & -i \sin (\Omega t / 2) \\
i \sin (\Omega t / 2) & \cos (\Omega t / 2)
\end{array}\right]\left[\begin{array}{l}
|\downarrow\rangle|\mathbf{0}\rangle \\
|\uparrow\rangle|\mathbf{1}\rangle
\end{array}\right](0),
$$

The ensemble displays Rabi-oscillations with an effective Rabi rate:

$$
\Omega=\sqrt{2 I_{0} \sum_{j}\left|a_{j}\right|^{2}} .
$$

At the same time, the spin-up electronic state $|\uparrow\rangle_{e} \otimes|\mathbf{0}\rangle_{n}$ is an eigenstate of both $\hat{V}_{D}$ and $V_{\Omega}$. Hence, by pulsing the applied field to go from $g^{*} \mu_{B} \hat{B}_{\text {eff }} \gg \hat{V}_{\Omega}$ to $\hat{B}_{\text {eff }} \sim 0$ for a time $t=\pi / \Omega$ as diagramed in Fig. प(b), an arbitrary superposition of electronic state will undergo the following evolution:

$$
\left(\alpha|\uparrow\rangle_{e}+\beta|\downarrow\rangle_{e}\right) \otimes|\mathbf{0}\rangle_{n} \rightarrow|\uparrow\rangle_{e} \otimes\left(\alpha|\mathbf{0}\rangle_{n}+i \beta|\mathbf{1}\rangle_{n}\right),
$$

demonstrating that an electronic spin state can be coherently mapped into nuclei.

The Rabi-flopping process can be controlled by removing the electron from the dot or by changing the effective magnetic field, $B_{0}$. Away from the resonance condition $\left(\left|B_{\text {eff }}\right| \gg \Omega / g^{*} \mu_{B}\right)$, the system is far detuned and no evolution will occur. For the perfectly polarized state the effective detuning is $\delta=\left(g^{*} \mu_{B}-\right.$ $\left.g_{n} \mu_{n}\right) B_{0}+I_{0} A+\left(I_{0}-1\right) A / N$. The Rabi frequency depends upon the distribution of the $a_{j}$ coefficients,
$\Omega=\sqrt{2 I_{0}} A / \sqrt{N}\left(1+\left(\Delta \bar{a}^{2}\right) / \bar{a}^{2}\right)^{1 / 2}$ with bars denoting averages over the set $\left\{a_{j}\right\}$. For a GaAs dot with $10^{4}$ nuclei and $\Delta^{-} a^{2} \sim \bar{a}^{2}, A \approx 90 \mu \mathrm{eV}$ and $I_{0}=3 / 2$, and the speed of transfer is determined by $\Omega / 2 \pi \simeq 0.6 \mathrm{GHz}$, which exceeds the expected spin coherence time by three orders of magnitude. The resonance condition is fulfilled for $\left|B_{\text {eff }}\right| \ll 50 \mathrm{mT}$. Retrieval of the stored qubit can be implemented by reversing this process: we either inject a spin-polarized electron into the dot or change the effective magnetic field, bringing the levels into resonance, and Rabi oscillations pick up at the same point as before.

Before proceeding with a detailed description of the coherence properties and imperfections we note that the above results can be easily generalized to the preparation of complex collective nuclear states. For example, injection of a series of spin-down electrons into spin-up polarized nuclei will lead to a progressive increase of the nuclear spin. In the basis of collective angular momentum, $\hat{\vec{I}}=\sum_{i} \hat{\overrightarrow{I^{i}}}$, we define the total angular momentum nuclear states $|\mathbf{m}\rangle_{n}=\left|I=N I_{0}, I_{z}=I-m\right\rangle_{n}$. Each electron can effect the transfer $|\downarrow\rangle_{e} \otimes|\mathbf{m}\rangle_{n} \rightarrow|\uparrow\rangle_{e} \otimes|\mathbf{m}+\mathbf{1}\rangle_{n}$. When injected electrons are prepared in different superposition states this process can be used to effectively "engineer" collective states of nuclear ensembles. In fact using a proper sequence of electrons an arbitrary state of the type $|\boldsymbol{\Psi}\rangle_{n}=\sum_{m=0}^{I} c_{m}|\mathbf{m}\rangle_{n}$ can be prepared [10]. We note in particular that the highly entangled states of the kind $\left(|\mathbf{0}\rangle_{n}+|\mathbf{m}\rangle_{n}\right) / \sqrt{2}$, with large $\mathbf{m}$ could be used for high-resolution NMR spectroscopy in analogy with related atomic physics studies [11, 12]. Such states can also be prepared by manipulating one electron in the dot with fast electron-spin resonance (ESR) pulses.

Injection of polarized electrons, combined with ESR pulses, can also be used to perform manipulation of the stored nuclear state. For example, the qubit stored in nuclear spin could be mapped into the injected electron and an ESR pulse can be used to rotate it. Subsequently, it can be mapped back to nuclei. Alternatively virtual, off-resonant $(|\delta| \gg \Omega)$ coupling of storage states to electron spin can be used to shift the resonance frequencies of transitions $|\mathbf{m}\rangle_{n} \rightarrow|\mathbf{m}+\mathbf{1}\rangle_{n}$ relative to each other. For example in the case of two lowest states $m=1,2$ the differential shift is on the order of $\Omega^{4} /|\delta|^{3}$. Whenever this shift is large compared to decoherence rate and the spectral width of excitation pulse, the lowest two states of collective manifold $|\mathbf{0}\rangle_{n},|\mathbf{1}\rangle_{n}$ can be considered as an effective two level system and can be manipulated through NMR pulses and other means. These ideas could be extended to the spin-exchange coupled qubits proposal [2].

We now turn to the consideration of various decoherence mechanisms and imperfections that limit the performance of the storage technique. To evaluate the effects of spatial inhomogeneity we note that the collective state $|\uparrow\rangle_{e} \otimes|1\rangle_{n}$ is not an eigenstate of $\hat{V}_{D}$ unless the $a_{j}$ 's are identical. The effect is determined by 
the distribution of eigenenergies under $\hat{V}_{D}$ of the states $|\uparrow\rangle_{e} \otimes\left|I_{0}, \ldots, I_{0}-1_{j}, \ldots, I_{0}\right\rangle_{n}$, given by

$$
E_{j}=\left(g^{*} \mu_{B} / 2-g_{N} \mu_{N} I_{0}\right) B_{0}+I_{0} A / 2-a_{j} / 2
$$

Since $\Delta \bar{E}^{2} \sim \Delta \bar{a}^{2}$, the inhomogeneous linewidth is $\sim$ $\bar{a}=A / N$. Hence inhomogeneous broadening is smaller than the relevant time scale for Rabi-flopping by a factor of $\sqrt{N}$, and is negligible during transfer operation. After the mapping, its effect can be mitigated by either removing the electron from the dot, thereby turning off the hyperfine interaction entirely, or by using ESR spin-echo techniques to reverse the phase evolution 13.

The leading decoherence process for the stored state is nuclear-spin diffusion, with dephasing rates in the $\mathrm{kHz}$ domain. However, techniques from NMR can be used to mitigate this effect 14, 15. By applying fast NMR pulse sequences [16] to rotate the nuclear spins the time average of the leading terms in the dipole-dipole Hamiltonian can be reduced to zero, leaving only residual dephasing due to second-order dipolar effects, pulse imperfections and terms due to the finite length of the averaging sequence. These phenomena have been studed for several decades for solid-state NMR systems and well-developed techniques routinely reduce $T_{2}$ by several orders of magnitude [14], down to sub-Hz levels. Hence, coherent qubit storage on the time scale of seconds seems feasible.

To evaluate the effects of partial polarization on the storage fidelity, we use the Heisenberg picture. In the homogeneous case $\left(a_{i}=a=A / N\right)$, the Dicke-like collective operators defined above yield $\hat{I}^{2}$ and $\hat{J}_{z}=\hat{S}_{z}+\hat{I}_{z}$ as the constants of motion. We consider operator equations of motion $\dot{\hat{A}}=i[\hat{A}, \hat{H}]$, for the three operators $\hat{S}_{z}, \hat{S}_{+} \hat{I}_{-}$, and $\hat{S}_{-} \hat{I}_{+}$, which commute with the constants of motion. We replace $\hat{I}_{z}$ terms in the resulting equations with $\hat{J}_{z}-\hat{S}_{z}$ and use the identity $\hat{I}_{+} \hat{I}_{-}=\hat{I}^{2}-\hat{J}_{z}^{2}-\left[\hat{I}_{-}, \hat{I}_{+}\right] / 2$ to put the equations in terms of constants of motion and the three operators we look to solve:

$$
\begin{aligned}
\frac{d}{d t} \hat{S}_{z} & =a \frac{\hat{S}_{+} \hat{I}_{-}-\left(\hat{S}_{+} \hat{I}_{-}\right)^{\dagger}}{2 i} \\
\frac{d}{d t}\left(\hat{S}_{+} \hat{I}_{-}\right) & =i\left[\left(g^{*} \mu_{B}-g_{n} \mu_{n}\right) B_{0}+a\left(\hat{J}_{z}-1\right)\right]\left(\hat{S}_{+} \hat{I}_{-}\right) \\
& -i a\left(\hat{I}^{2}-\hat{J}_{z}^{2}+1 / 4\right) \hat{S}_{z}
\end{aligned}
$$

It is convenient to choose new constants of motion, $\hat{\delta}=$ $\left(\left(g^{*} \mu_{B}-g_{n} \mu_{n}\right) B_{0}+a\left(\hat{J}_{z}-1\right)\right)$ and $\hat{\Omega}=a\left(\hat{I}^{2}-\hat{J}_{z}^{2}+1 / 4\right)^{1 / 2}$. These commute with each other and with $\hat{S}_{z}, \hat{S}_{+} \hat{I}_{-}$. As these equations are similar to those for two-level atoms in a field, we make the Bloch vector identifications:

$$
\begin{aligned}
\hat{U} & =-a / \hat{\Omega} \frac{\hat{S}_{+} \hat{I}_{-}+\left(\hat{S}_{+} \hat{I}_{-}\right)^{\dagger}}{2}, \\
\hat{V} & =-a / \hat{\Omega} \frac{\hat{S}_{+} \hat{I}_{-}-\left(\hat{S}_{+} \hat{I}_{-}\right)^{\dagger}}{2 i}, \\
\hat{W} & =\hat{S}_{z} .
\end{aligned}
$$
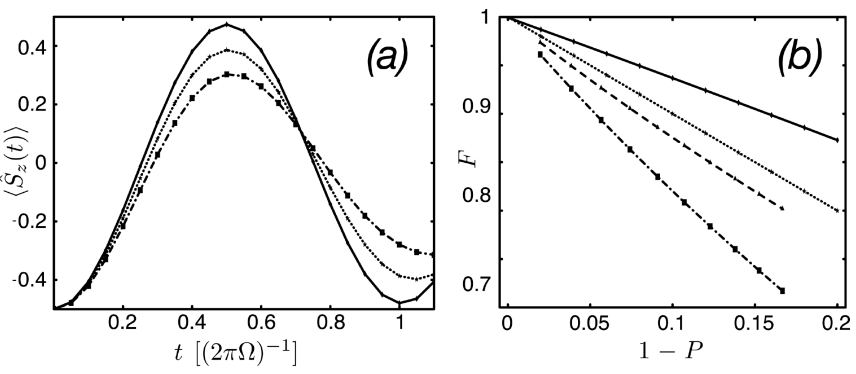

FIG. 2: Transfer characteristics for different nuclear polarizations. (a) The evolution of $\left\langle S_{z}(t)\right\rangle$ for three nuclear polarizations, $P=0.96$ (solid curve), 0.82 (dotted), and 0.67 (dotdash) versus time in units of the $P=1$ Rabi time. (b) Solid line: Storage fidelity $F$ for inhomogeneous effects $\left(N=10^{4}\right)$ versus error in nuclear polarization, $1-P$. Dotted line: analytical estimate of thermal effects. Dashed line: numerical simulations of thermal effects. Dot-dash line: product of inhomogeneous and thermal results.

and Eqns. 3-4 become $\dot{\overrightarrow{\vec{M}}}=\hat{\vec{M}} \times \hat{\overrightarrow{\vec{\omega}}}$ with $\hat{\vec{\omega}}=(\hat{\Omega}, 0,-\hat{\delta})$. The Bloch vector operator, $\hat{\vec{M}}$, will rotate about the axis defined by $\hat{\vec{\omega}}$ at a frequency $\hat{\omega}_{0}=\sqrt{\hat{\delta}^{2}+\hat{\Omega}^{2}}$. For no initial electron spin-nuclear spin correlation, we can easily solve for $\hat{S}_{z}$ and find

$$
\left\langle\hat{S}_{z}(t)\right\rangle=\left\langle\frac{\hat{\delta}^{2}+\hat{\Omega}^{2} \cos \left(\hat{\omega}_{0} t\right)}{\hat{\omega}_{0}^{2}} S_{z}(0)\right\rangle .
$$

For the perfectly polarized nuclear state, Eq. 6 gives $\left\langle\hat{S}_{z}\right\rangle=-1 / 2 \cos \left(A \sqrt{2 I_{0} / N} t\right)$ for the spin-down species and $1 / 2$ for the spin-up species, exactly replicating the fully polarized behavior. In the case of partial polarization, even though we will set $\langle\hat{\delta}\rangle=0,\left\langle\hat{\delta}^{2}\right\rangle$ remains finite. The first part of Eq. 6] prevents complete transfer, to order $\left\langle\hat{\delta}^{2}\right\rangle /\left\langle\hat{\omega}_{0}^{2}\right\rangle$. For partial polarization $P<1$, $\left\langle\hat{\omega}_{0}^{2}\right\rangle \approx a^{2} N\left(2 I_{0}+O(1-P)\right)$. In a thermal state, all 2-particle expectation values factor, so $\langle\hat{\delta}\rangle=0$ and

$$
\left\langle\hat{\delta}^{2}\right\rangle=a^{2}\left(\left\langle\hat{J}_{z}^{2}\right\rangle-\left\langle\hat{J}_{z}\right\rangle^{2}\right) \approx N a^{2}(1-P) 2 I_{0} .
$$

Accordingly, the error scales as

$$
\frac{\left\langle\hat{\delta}^{2}\right\rangle}{\left\langle\hat{\omega}_{0}^{2}\right\rangle} \approx 1-P .
$$

Remarkably, this result demonstrates that efficient transfer is possible even with many nuclei in the "wrong" state as long as the average polarization per nuclei is high.

We modeled this system numerically with an initial thermal nuclear state. Oscillation of $\left\langle\hat{S}_{z}\right\rangle$ for several polarizations is shown in Fig. 2(a). The effect of partial polarization is immediately apparent; the transfer peak is less than $1 / 2$, and the Rabi frequency decreases. The difference between the initial electron state and the state after once cycle of storage and retreival corresponds to the storage fidelity $F$. Fig. 2(b) shows that the analytical estimate is close to the numerically calculated 
values; thus, the transfer error can, to a large extent, be explained by the thermal uncertainty in $\hat{\delta}$. The residual effect most likely stems from phase mismatching, as measured by the broadening of $\omega_{0}$, such that at $t=\left(\pi\left\langle\omega_{0}\right\rangle\right)^{-1},\left\langle\cos \left(\omega_{0} t\right)\right\rangle=1-O(1-P)$.

Inhomogeneous broadening for a thermal initial nuclear state is somewhat more pronounced than the fully polarized case first considered. The $\hat{V}_{D}$ inhomogeneity causes slow dephasing of the stored state, and the inhomogeneous coupling in $\hat{V}_{\Omega}$ results in leakage in readout of the stored state into a set of states orthogonal to the original nuclear state. The results shown in Fig. 2(b) were calculated for a distribution of $a_{i}$ 's corresponding to a gaussian $|\psi(\vec{r})|^{2}$. We plot the estimated fidelity for a complete storage and readout cycle as a function of the initial polarization of nuclear spins. The total expected fidelity, is approximated by the product of these two results (Fig. 2(b)). Hence, only modest nuclear polarizations are required to obtain a high fidelity of storage.

In summary, we have demonstrated that is possible to reliably map the quantum state of a spin qubit onto long-lived collective nuclear spin states. The resulting states have long coherence times, and can be retrieved on demand. Furthermore, the stored states can be efficiently manipulated and similar techniques can be used for quantum state engineering of collective nuclear states.

Experimental implementation of these ideas requires preparation of nuclear spin polarizations in the vicinity of confined electrons. Optical pumping of nuclear spins has demonstrated polarizations in GaAs 2D electron gases on the order of $90 \%$, 17] and $65 \%$ in self-assembled dots [18], and forced spin-flips through quantum hall edge states [19] has a claim of a similarly high polarization ability (85\%). We anticipate that combining either of these techniques with electron localization in quantum dots would be a promising avenue of study. The methods outlined in this paper can be used to further increase the nuclear polarization. A current of spin-polarized electrons passing through the quantum dot with a dwell time $\tau_{\text {dwell }}<\pi / \Omega_{0}$ will result in spin transfer, thereby increasing nuclear polarization. By keeping $B_{\text {eff }}$ tuned to zero with increasing nuclear polarization the spin flip-flop remains resonant and, when combined with dephasing to prevent saturation, leads to efficient cooling, similar to a recent proposal [20].

Coherence properties of the spin-exchange process could be probed in transport measurements. For example, sending spin polarized currents through the quantum dot in which the spin-exchange interaction is tuned to resonance will result in collapses and revivals of the electron spin polarization that will be a periodic function of the dwell time in the dot. Those can be measured using spin-filter techniques. For a given polarization $P$ and $\tau_{\text {dwell }}$, the spin will be rotated by $\Omega(P) \tau_{\text {dwell }} / \pi$. Note that repolarization will be necessary after $\sim \sqrt{N}$ nuclear spins have been flipped.

Practical applications of the storage and retrieval techniques and manipulation of stored states requires timevarying control over the spin-exchange coupling. This can be accomplished by using a pulsed magnetic field of order $50 \mathrm{mT}$ for a few ns, by engineering the electron $g$ factors [21, 22], or by optical AC Stark shifts 17] These techniques can be combined with a number of avenues for entanglement and manipulation of the electronic spin and charge states currently under exploration 1, 2]. Finally, quantum memory can facilitate implementation and reduce scaling problems for more ambitious tasks such as quantum error correction [23] or quantum repeaters [9].

We thank J.A. Folk, Y. Yamamoto, and D. Loss for helpful discussions. This work was supported by ARO, NSF, and by David and Lucille Packard Foundation.

[1] D. D. Awschalom, N. Samarth, and D. Loss, eds., Semiconductor Spintronics and Quantum Computation (Springer-Verlag, Berlin, 2002).

[2] D. Loss and D. DiVincenzo, Phys. Rev. A 57, 120 (1998).

[3] G. Burkard, H.-A. Engel, and D. Loss, Fortschr. Phys 48, 965 (2000).

[4] T. Fujisawa, Y. Tokura, and Y. Hirayama, Phys. Rev. B. 63, R081304 (2001).

[5] B. Kane, Nature 393, 133 (1998).

[6] A. Khaetskii, D. Loss, and L. Glazman, Phys. Rev. Lett. 88, 186802 (2002).

[7] I. A. Merkulov, Al. L. Efros, and M. Rosen, Phys. Rev. B 65, 205309 (2002).

[8] A. Khaetskii, D. Loss, and L. Glazman, condmat/0211678 (2002).

[9] L. Duan, M. D. Lukin, J. I. Cirac, and P. Zoller, Nature 414, 413 (2001).

[10] For spin-1/2 nuclei considering a reverse process $|\Psi\rangle \rightarrow$ $|\mathbf{0}\rangle$ provides a proof, in direct analogy to C.K. Law and J.H. Eberly, Phys. Rev. Lett. 76, 1055 (1996). The same argument applies to larger nuclear spin $I_{0}$ when $\mathbf{m} \ll I$.

[11] J. Bollinger et al., Phys. Rev. A 54, 4649 (1996).

[12] S. Huelga et al., Phys. Rev. Lett. 79, 3865 (1997).

[13] H. Carr and E. Purcell, Phys. Rev. 80, 580 (1954).

[14] M. Mehring, High Resolution NMR Spectroscopy in Solids (Spring-Verlag, Berlin, 1976).

[15] D. Paget, G. Lampel, B. Sapoval, and V. Safarov, Phys. Rev. B 15, 5780 (1977).

[16] J. Waugh, L. Huber, and U. Haeberlen, Phys. Rev. Lett. 20, 180 (1968).

[17] G. Salis, D. D. Awschalom, Y. Ohno, and H. Ohno, Phys. Rev. B 64, R195304 (2001).

[18] D. Gammon et al., Phys. Rev. Lett. 86, 5176 (2001).

[19] D. Dixon, K. Wald, P. McEuen, and M. Melloch, Phys. Rev. B 56, 4743 (1997).

[20] M. Eto, cond-mat/0210231 (2002).

[21] G. Salis et al., Nature 414, 619 (2001).

[22] K. A. Matveev, L. I. Glazman, and A. I. Larkin, Phys. Rev. Lett. 85, 2789 (2000).

[23] A. Steane, Nature 399, 124 (1999). 